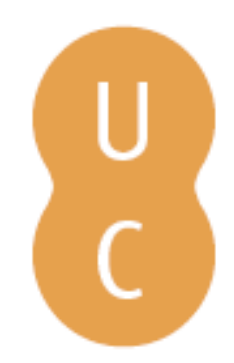

\title{
pompalina
}

\section{Hechos y pensamientos sobre educación en busca de una teoría: la trama entre Biología, Tecnología y Cultura}

\author{
Autor(es): $\quad$ García del Dujo, Ángel; García Carrasco, Joaquín; Asensio Aguilera, \\ José María
}

Publicado por: Imprensa da Universidade de Coimbra

URL persistente:

URI:http://hdl.handle.net/10316.2/38384

DOI:

DOI:http://dx.doi.org/10.14195/978-989-26-0486-2_6

Accessed : $\quad$ 26-Apr-2023 13:05:11

A navegação consulta e descarregamento dos títulos inseridos nas Bibliotecas Digitais UC Digitalis, UC Pombalina e UC Impactum, pressupõem a aceitação plena e sem reservas dos Termos e Condições de Uso destas Bibliotecas Digitais, disponíveis em https://digitalis.uc.pt/pt-pt/termos.

Conforme exposto nos referidos Termos e Condições de Uso, o descarregamento de títulos de acesso restrito requer uma licença válida de autorização devendo o utilizador aceder ao(s) documento(s) a partir de um endereço de IP da instituição detentora da supramencionada licença.

Ao utilizador é apenas permitido o descarregamento para uso pessoal, pelo que o emprego do(s) título(s) descarregado(s) para outro fim, designadamente comercial, carece de autorização do respetivo autor ou editor da obra.

Na medida em que todas as obras da UC Digitalis se encontram protegidas pelo Código do Direito de Autor e Direitos Conexos e demais legislação aplicável, toda a cópia, parcial ou total, deste documento, nos casos em que é legalmente admitida, deverá conter ou fazer-se acompanhar por este aviso.

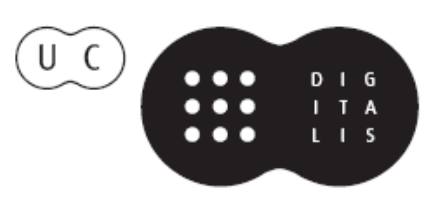


João Boavida

Ángel García del Dujo

Coordenação

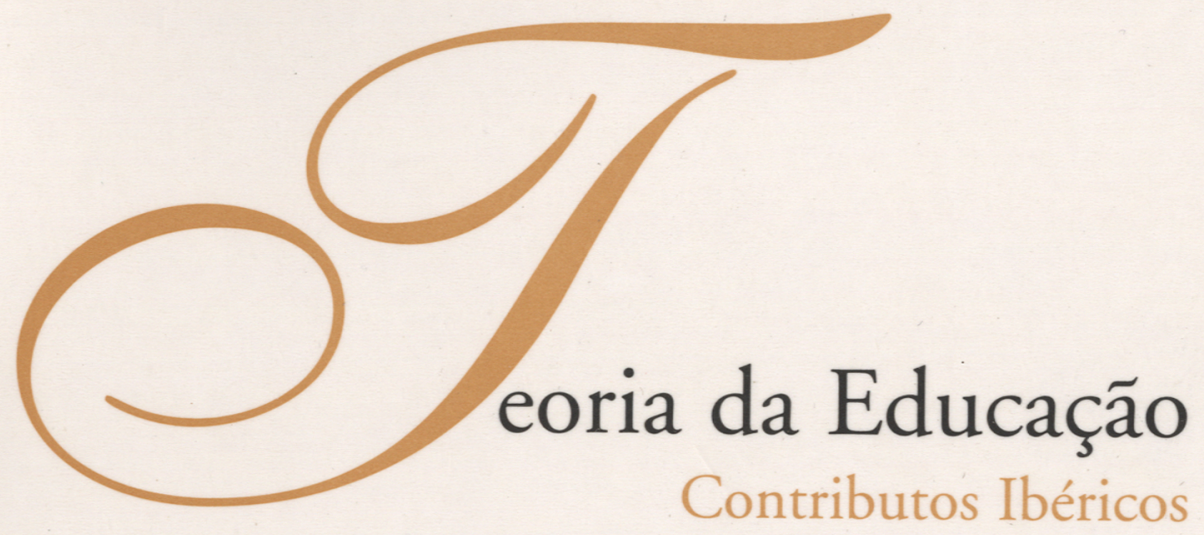


Ángel García del Dujo (coordinador)

Joaquín García Carrasco

Universid de Salamanca

José M aría A sensio A guilera

Universid Autónoma de Barcelona

Hechos y Pensamient os sobre Educación en busca de una

Teoría: La trama entre Biol ogía, Tecnol ogía y Cul tura

\section{Aclaraciones iniciales prescindibles...}

El título contiene una velada alusión a la obra teatral de Luigi Pirandello, Sei personaggi in cerca d'autore, donde el autor lleva a cabo un ejercicio, teórico y empírico, de reflexión sobre la forma de plantear los componentes, estructura y naturaleza del teatro. La razón de esta referencia es muy sencilla: entendemos que lo que se nos está pidiendo en este artículo es, en última instancia, un ejercicio de reflexión sobre los modos como nosotros venimos planteando, pensando y haciendo, Teoría de la Educación; y, puesto que creemos que también en este campo, en estos momentos, hay «personajes» - hechos, fenómenos y resultados de investigación - que estarían reclamando su entrada en escena, con repercusiones importantes en la forma de abordar el estudio de los fenómenos educativos, hemos creído oportuno titular esta reflexión con una paráfrasis de una obra reconocida por todos como fundamental en la evolución del teatro del siglo XX. Quizá estemos, salvando todas las distancias necesarias, ante una situación análoga, eso sí, en el caso de que «nuestros personajes» sean admitidos definitivamente al escenario donde «se representan» los fenómenos educativos. 
De manera más explícita, este trabajo pretende concretar y clarificar la siguiente posición: en nuestra investigación de la última década venimos encontrándonos reiteradamente con algunos hechos, datos en otras ocasiones, indicios la mayoría de las veces, sobre el proceso de conformación del ser humano que estarían aconsejando una relectura de algunos fundamentos, orientaciones y principios explicativos del fenómeno educativo, con repercusiones también, y no menores, en los modos de pensar y hacer hoy Teoría de la Educación.

En este caso, estamos pensando en tres campos temáticos cuyas aportaciones individuales no pueden hoy ser ignoradas, mucho menos si cruzamos la información que cada uno de ellos nos proporciona; por eso, podríamos expresar ahora nuestra pretensión en los siguientes términos: tres núcleos temáticos a la búsqueda de integración en un sistema teórico.

Esos tres núcleos temáticos — por supuesto, no son los únicos que aportan conocimientos de interés para el análisis de la educación —on referenciados aquí mediante tres términos de sencilla ubicación en la geometría del conocimiento: la vida, la técnica y la cultura; en rigor, nuestro trabajo intenta reubicar los dos primeros; el tercero, estaría señalando su destino, pero sin su enunciación quedaría incompleto nuestro planteamiento.

Hay, todavía, en nuestra propuesta otro supuesto, que aceptamos y queremos hacer explícito: pareciera que la investigación sobre la educación se mueve entre varias perspectivas, sin duda complementarias, a la búsqueda de integración. En este trabajo nos concentramos en dos de ellas, por considerarlas los autores de particular importancia en el momento actual, porque ambas forman parte de nuestras líneas de trabajo $y$, en última instancia, porque las dos resultan imprescindibles, mejor dicho, aportan componentes imprescindibles para tejer la trama sobre la que se asienta la inevitable condición del ser humano, su condición cultural. Y todo ello, no precisamente porque sea éste el objetivo de la educación 0 el objeto de la Teoría de la Educación, sino su inexcusable punto de apoyo y de partida. 


\section{2. ... e imprescindibles}

El primer componente y término de la trama (biología) está reconociendo la necesidad que tiene el pensamiento sobre la formación de integrar el conocimiento del mundo de la vida (García Carrasco y García del Dujo, 2001), por varias razones estrechamente relacionadas: a) la necesidad de una teoría de la educación que parta de la unidad psicosomática y contemple la condición humana en toda su dinámica y manifestaciones, pues en ella está instalada también la imperfección, la discapacidad, el deterioro físico y mental, la enfermedad y la muerte, al tiempo que se mueve por el afecto, busca el bienestar y se encuentra indefectiblemente asociada al cuerpo; b) la necesidad de una teoría de la educación que rompa con algunas discontinuidades construidas entre los seres humanos y el resto de los seres vivos, en cuanto que están dificultando notablemente la interpretación y el tratamiento de una educación más amplia y diversa y c) la necesidad de una teoría de la educación que integre en su reflexión la deliberación sobre el progreso sostenible, ya que el estado que presenta en la actualidad la condición cultural del ser humano afronta la responsabilidad de preservar la biosfera como condición para el mantenimiento de la calidad de vida posible en futuras generaciones.

Nada de lo anterior se justifica adecuadamente si no se explicita dentro de un humanismo construido desde la pertenencia al mundo de la vida, donde la comprensión de la vida y la comprensión de la condición humana se apoyan mutuamente. En el fondo, estamos replanteando las relaciones entre vida y formación, desde la insatisfacción del habitual modo de entender las de la naturaleza y la cultura. Estas relaciones han sido controvertidas, muchas veces han estado contrapuestas, y están sometidas a debate constante desde la Ilustración.

El segundo componente y término de la trama (tecnología) trae hoy inmediatamente a la memoria la contingencia cultural en la que se encuentra sumida la sociedad contemporánea, pero la razón de su presencia en la trama es mucho más sólida y anterior. 
El amplio abanico de posibilidades que en estos momentos nos ofrece la nueva condición tecnológica no debe hacernos olvidar que la relación entre tecnología y construcción de los procesos sociales, evidente en diversos momentos históricos (Briggs y Burke, 2000) fue y es uno de los mecanismos constituyentes del ser humano y la forma como el sujeto y la sociedad se reinventan constantemente, hasta el punto de llegar a pensar que «la cultura no existe, no sobrevive, si no es en un medio progresivamente más sofisticado tecnológicamente»(Broncano, 1995, 10). Ni la cultura ni, lo que es más importante para nosotros, la condición cultural del ser humano, donde descansa la tarea educativa.

A la contingencia cultural actual, referenciada por el concepto de Sociedad de la Información y otros términos equivalentes y/0 complementarios, está contribuyendo el desarrollo tecnológico en los ámbitos de la comunicación, la producción y la gestión de la información, así como el diseño de herramientas capaces de realizar complejas operaciones, que simulan y hasta emulan la capacidad realizativa humana 0 el alcance funcional de algunas de sus aptitudes mentales, y con posibilidades incluso de generar «otros escenarios» de acción.

Ello obliga a reconsiderar el status de todo tipo de instrumentos herramientas, artificios, artefactos, instrumentos psicológicos —en vistas a comprender el alcance real de su papel de mediación en las prácticas formativas, en cuanto que lo está significando en la práctica cultural. En síntesis, replanteamos el modo de considerar las técnicas y las tecnologías humanas, por estimar insatisfactorias las habituales contraposiciones entre el modelo técnico y el modelo histórico social — con esta u otras denominaciones — en los discursos sobre la educación; más aún, por estimar pernicioso para la educación «the dualism that not only separates things from persons and qualitatively differentiates them, but that privileges the social over the material and technological. Of course, such a dualism also invites the inverse» (Waltz, 2002).

Del tercer término (cultura) decíamos antes que resulta imprescindible en nuestro planteamiento en cuanto que es propiamente la trama sobre la 
que se asienta la posibilidad y necesidad formativa del ser humano. Quiere esto decir que estamos entendiendo este término en su significado primario (García Carrasco y García del Dujo, 1997), no tanto como cultura objetiva cuanto la posibilidad y la práctica de la incorporación cultural, que en su evolución y desarrollo llevarán a la calidad de las operaciones mentales implicadas en las prácticas de formación.

La capacidad de construir creencias acerca de los estados mentales del otro permite diseñar y promover prácticas comunicativas, integradas por mediaciones de todo tipo, a través de las cuales la cultura es incorporada; esas funciones mentales y esas mediaciones hacen posible la cooperación significativa en las prácticas, así como la configuración de funciones mentales superiores 0 de progresiva elaboración, complejidad y abstracción. Las aptitudes mentales humanas permiten que la participación en la práctica se traduzca en aprendizaje significativo, proporcionando, al mismo tiempo, oportunidad para la deliberación sobre el sentido y el valor moral de la práctica. La creación cultural consuma su significado en la medida en que es comunitariamente incorporada, en forma de conocimiento del mundo de la vida, de competencia para la creación de calidad de vida y de comprensión de la dialéctica entre ambos componentes.

De ahí se deriva que la Pedagogía no completa su marco de referencia teórico si lo construye únicamente desde un contexto de procedimientos; la función de incorporación cultural hace referencia insoslayable a los contenidos y valores. Dentro de la responsabilidad de la formación hay una inaplazable tarea de comprensión e integración, mediante la cual se disponen los conocimientos disponibles en unidades temáticas accesibles, en vistas a su incorporación cultural significativa. La Pedagogía, además de la investigación procedimental, tiene necesidad de implicarse en el ejercicio de la comprensión cultural; no sólo en la comprensión de las prácticas de la formación, sino también en las prácticas de comprensión del mundo y de la vida; por esta vía irá hacia la comprensión de la educación, como elemento básico del modo humano de vida. 
En la comprensión, reclamada por la formación, para comprender y comprenderse, juegan hoy un papel fundamental las cuestiones relacionadas con la vida y las cuestiones relacionadas con la tecnología. La formación brega con el acervo cultural disponible, pero también con problemas no resueltos, cultura insostenible, y con situaciones de cultura indisponible. La reflexión sobre la formación afronta cuestiones de incorporación cultural no discutibles; afronta también la formación del pensamiento crítico frente a situaciones civilizatorias injustificables y ha de hacerlo, también, con la necesidad de cooperar en la deliberación responsable sobre la vida, porque tampoco es discutible que dentro del mundo de la vida tiene lugar el conocimiento y la conciencia. La Pedagogía adquiere, pues, un claro compromiso teórico, junto al indiscutible compromiso práctico. El compromiso teórico no puede reducirse al de la investigación y la reflexión en la acción, sino que ha de ampliarse a la investigación y la reflexión sobre los componentes, contenidos y valores clave de la cultura. Precisamente éste es el problema: entendemos que en la cultura adquieren una relevancia esencial la comprensión de la vida y la comprensión de las mediaciones instrumentales. Ambas perspectivas de interés primordial para nosotros: lo primero, porque de ahí emana en buena parte la representación del ser humano sobre la que se construye la teoría de la educación; lo segundo, porque la comprensión de las prácticas formativas gravita sobre su condición de acción mediada, acción social e instrumentalmente mediada.

\section{Relectura de la (teoría de la) educación desde el conocimiento de la vida}

El interés que tiene la Teoría de la Educación en la comprensión de la vida no exige que el pedagogo sea experto en Biología, ni se colma la necesidad mediante el sencillo recurso a los materiales sistemáticos que este campo disciplinar aporta. La relectura ha de llevarse a cabo mediante la orientación 
que proporciona una pregunta vigorosa: cuáles son las posibilidades y los límites de la condición educable del ser humano (Asensio, 1997).

La educación ha sido leída desde la advertencia de que el proceso cultural está históricamente condicionado; también to ha sido desde la experiencia de que el proceso de formación está socialmente mediado. La relectura biológica de la educación debe partir de la consideración de que la formación es vitalmente necesaria y del hecho de que se encuentra biológicamente condicionada. La meta de la investigación es conseguir la comprensión de tal necesidad vital y de ese condicionamiento biológico. El trabajo riguroso de la Teoría de la Educación consiste en construir ese edificio de significados, que remediaría, por otra parte, la precaria situación actual en que se encuentra en este punto la formación de los pedagogos en nuestras Facultades de Educación.

Pero, antes de adentrarnos en esta perspectiva, vamos a trazar, aunque sea con grandes rasgos, lo que bien podría llamarse, en la cultura occidental, enfoque dominante en la tradición reflexiva sobre el mundo de la vida; conviene que tengamos delante las grandes líneas de esa reflexión.

Ese enfoque, por lo menos hasta el Renacimiento, podría resumirse diciendo que contenía una antropología de dominancia -el hombre en la cumbre de la jerarquía de los seres vivos —que se completaba con la visión de que la Tierra era el centro del Universo. La perspectiva antropocéntrica era global, coherente: la Tierra en el centro del Cosmos, el hombre en el centro de la Tierra, desde donde se origina el significado de toda forma de vida aquí en la Tierra.

El primer centro de perspectiva se resquebraja cuando Copérnico comienza en 1503 su tratado De revolutionibus orbium celestium el segundo se viene abajo cuando Darwin, consciente de las consecuencias, edita en 1896 Maris Place in Nature. La filosofía occidental era consciente de lo que estaba pasando; por eso, El puesto del hombre en el Cosmos también fue el título de una obra de Max Scheler publicada en 1928, revisando las relaciones entre naturaleza y cultura. 
Dentro de esta historia de confrontaciones entre naturaleza y cultura, de empeños por mantener la condición humana sobrepuesta respecto del mundo de los seres vivos y de ir admitiendo las evidencias evolutivas, aparecieron dos fuentes de perspectiva desde la que entender las acciones de formación, construidas ambas desde lo que denominaríamos una antropología de la exclusividad, una antropología elaborada desde los caracteres que segregan la condición humana del resto del mundo de la vida: la cultura sobreponiéndose a toda forma de comportamiento en la naturaleza y la mente sobreponiéndose, a su vez, a toda forma 0 manifestación de corporeidad.

Del primer foco de la perspectiva, en lo que a educación se refiere, el epígono fue J. Locke y del segundo el artífice que recibió mayor reconocimiento fue Descartes. Wertsch coincide en señalar que la perspectiva que considera el ser humano como el receptor de incitaciones, indicaciones e influencias ambientales, respecto a las cuales su bios está a la expectativa y como página en blanco, lleva sus raíces hasta la autoridad de Locke, quien se suma al empirismo de Hobbes y a su analogía de la «tabula rasa». Locke concreta su sistemática sobre el origen de las ideas en su famoso Ensayo sobre el entendimiento humano. Descartes, por su parte, plantea el ambiente como mero inductor para desencadenar procesos en la realidad pensante situada en el interior del individuo particular. Dos consecuencias añadidas llevaba la perspectiva: el sujeto individual se tomará como el «locus» de la realidad y el cambio en el pensamiento y las actividades del cuerpo podrán ser tratadas como las propias de una «pieza de intrincada maquinaria», cuya analogía preferente será la de un reloj (Smith, 1977).

El meollo de la perspectiva parece inducir la creencia de que la mente puede concluir que ha comprendido cuando consigue construir el reloj o que su construcción es posible; a medida que profundiza en la complejidad de la máquina, consuma en el experimento la eliminación definitiva de la subjetividad. Tal vez aquí se encuentre el punto en el que comienza la profunda segregación entre naturaleza y cultura, entre ciencias de la naturaleza y ciencias del espíritu, la disgregación entre las acciones vitales en el mundo y la conformación 
de la mente en la cultura. Y tal vez el punto álgido de la confrontación llegó con la identificación de la mente con la máquina extremadamente compleja del cerebro (The Mind-Brain Identity Theory); de la segregación inicial brotó el materialismo más radical, que hoy de nuevo reverbera.

La perspectiva se siguió alimentando cuando Niels Bohr (1885-1954) advierte que los modelos de la conservación de los genes y las matemáticas de la herencia, que descubriera Mendel, presentaban semejanzas con los cálculos fundamentales de la teoría cuántica. Max Delbrück (1906-1981), su discípulo en Copenhague, interpretó los términos de la discontinuidad entre las formas de vida, las divergencias en los rasgos y las variaciones dentro de un rasgo, en términos cuánticos. A esta línea de investigación, argumentando en la misma perspectiva, se adhirió Edwin Schrõdinger al publicar en 1944 su influyente libro ¿Qué es la vida?, proponiendo una interpretación del mecanismo de la herencia como sistema mecánico cuántico y uniéndose a la idea de que las moléculas de ADN habrían de ser cristales aperiódicos. Todos entendieron que podríamos explicar la vida, incluso su ser-además, respecto a la piedra, sin salimos de las vigencias del mundo de la física. Sin embargo, los propios descubrimientos científicos demostraron que la perspectiva puramente física era incorrecta. Era una reducción: las leyes de la física aportaban una vigencia, dentro de la vigencia de la propia organización de la vida.

En 1953, Watson y Crieck demostraron que el ADN era una molécula estructuralmente estable y que su misma estructura hacía posible la consecuencia de la autorreplicación, su propiedad más característica (Davies, 2001). Cuando en el año 2000 se presenta el mapa avanzado del genoma humano, C. Venter, uno de sus artífices, entendió que «era un momento histórico en los 100.000 años de humanidad» y, dando tarea a las humanidades, añadió: «Las complejidades y las maravillas del proceso por el que los compuestos químicos inanimados que forman nuestro código genético dan lugar a los imponderables del espíritu humano deberían inspirar a los poetas y a los filósofos durante milenios» (p. 317). 
Pero, también la ciencia con sus descubrimientos vuelve a confirmar que la vigencia de las leyes de la física se mantiene dentro de la vigencia de las leyes de la biología y que, dentro de ambas, se sitúa la vigencia de la subjetividad. La comprensión del proceso de formación de una persona de nuestro tiempo debe ser hecha manteniendo el recorrido de todas esas vigencias.

Esto significa que el punto de partida y apoyo de la reorientación que necesitamos podría resumirse escuetamente diciendo que, lo que las convicciones mantuvieron y desearon mantener en planos separados, naturaleza y cultura, es inseparable. En esta línea contamos ya con varias propuestas de integración.

H. Joñas $(1995,1997)$ muestra cómo una antropología construida desde la exclusividad, caso de la antropología existencialista, «priva a la comprensión del mundo orgánico de los resultados que alcanza la autopercepción humana y por esa misma razón traza mal la verdadera línea divisoria entre el animal y el hombre»; por el contrario, «la biología científica, atada por sus propias reglas a los hechos físicos externos, se ve obligada a pasar por alto la dimensión de interioridad propia de la vida» (Joñas, 2000). Su propuesta es reflexionar la vida desde la unidad psicosomática, reflexionar el organismo como forma objetiva de vida, que se muestra y autointerpreta en la reflexión sobre el hombre. El mundo de la vida se presenta ante el hombre para admirar la gradación de capacidades con las que los organismos hacen frente a sus pretensiones: metabolismo, sensación, movimiento, emoción, percepción, imaginación y creación simbólica. Lo que el dualismo segregó, sin abolir ahora la polaridad, se asume y supera en una unidad superior, donde los polos han de aparecer como «aspectos de la realidad del ser 0 fases en su devenir».

Por su parte, varios biólogos están realizando un esfuerzo por plantear una comprensión global de la vida y su gradación, al tiempo que muestran la evolución de las aptitudes emergentes con las que cumple sus funciones vitales (Maynard-Smith y Szathmáry, 2001). Mediante este rastreo se intenta 
encontrar las condiciones básicas de toda forma de vida; en el proyecto se recupera, como proceso de la máxima relevancia, el momento inicial (Margulis y Sagan, 1996, 2001). Este procedimiento de encontrar la raíz de toda forma de vida se revela como una estrategia significativa desde la que encontrar el punto de unión entre el bios y el conocimiento (Maturana y Varela, 1990). En el fondo, se trata de estudiar la vida desde su propiedad fundamental, la autonomía (García Carrasco, 2002). Las organizaciones moleculares, en aquel momento inicial, adquirieron la propiedad de clausurarse respecto a todo lo demás; iniciaron un proceso circular y recursivo de auto-reproducción y auto-configuración y constituyeron los primeros organismos, las primeras células. Una de las funciones de ese proceso tenía la meta de producir un componente fundamental de su estructura: la membrana que cierra e identifica al organismo, diferencia la función comunicativa interior-exterior y confina un espacio de «subjetividad» respecto al entorno. Hacia el interior de la membrana tiene lugar el fenómeno de la identidad celular. Antes, el universo estaba formado únicamente por cosas; a partir de ese momento, se inició el mundo de los seres vivos y el hombre puede hablar de él mediante historias vitales. Aquella unidad vital, capaz de auto construirse, auto repararse y auto reproducirse, constituyó la forma primodial de «corporeidad», la primera apariencia corporal.

Humberto Maturana y Francisco Varela llamaron sistema autopoiético a las identidades orgánicas, al carácter de autoconstrucción clausurada 10 calificaron de autopoiesis. Aquel patrón de organización poseía la propiedad de auto regularse, funcionaba como una red de relaciones que se regulan a sí mismas, con capacidad de producir su propia réplica y de hacerlo de manera no absolutamente'fiel; es decir, en la misma estructura estaba contenido el principio de reproducción y los de variación y biodiversidad. En adelante, la evolución en la complejidad de la vida promoverá, al tiempo, la diversificación del sistema autopoiético, manteniendo aquellas propiedades primarias auto referenciales perfectamente reconocibles durante todo el recorrido evolutivo. 
Una integración teórica coherente entre los interrogantes de la formación y las aportaciones de la biología es vista por los estudiosos como necesaria, al tiempo que presenta dificultades no pequeñas, como la sospecha de una brecha semántica entre ambos dominios, lo que estaría dando a entender que existen problemas epistemológicos generales; esto es precisamente lo que constataron el bioneurólogo Changuex y el filósofo Ricoeur (1999) a propósito de la creación de un marco cognitivo compartido respecto a la actividad de pensamiento.

Las dificultades comienzan incluso por el hecho de compartir un mismo aparato conceptual. Un ejemplo concreto es el propio concepto de autopoiesis, introducido por Maturana y Varela en 1973. El marco general en el que el concepto se gesta es la convicción de los autores de las limitaciones de los conceptos de información y representación -promovido el uno por la cibernética y el otro por el programa cognitivista- para entender el sistema biológico (Varela, 1996). En su lugar, Maturana y Varela retoman otros de tradición filosófica —auto referencia y autonomía — como pivote esencial para la comprensión de la unidad celular. Ello tiene implicaciones respecto al propio concepto de representación, pues toma relevancia la concatenación interna de procesos en un sistema clausurado.

Piaget había publicado en 1969 Biología y conocimiento proponiendo una revisión de la perspectiva biológica en el sentido de subrayar «la autonomía del ser vivo». El concepto de autopoiesis ponía en relación los conceptos de autonomía, organización (patrón de interacción entre componentes), identidad orgánica, circularidad y procesos auto funcionales (auto construcción, auto reparación, auto organización). La teoría autopoiética unificada fue presentada en 1984, entendiendo sus promotores que esos fenómenos autopoiéticos inauguran los fenómenos interpretativos en cuanto que algunos rasgos del mundo son tomados por el organismo como señales para la regulación de sus funciones propias. En el progreso conceptual terminaron vinculándose, como patrones que recorren toda la historia evolutiva, las propiedades de autonomía y cognición (Varela, 1989), instituyendo así la posibilidad de 
tomar ambos conceptos dentro de una narrativa de tramas que conectan todo el mundo de la vida(1).

El concepto de autopoiesis fue bien aceptado, manteniéndose en el ámbito de la biología incluso como criterio para una narrativa global de la vida. Pero saltó al ámbito de las ciencias humanas. El concepto fue transferido, como metonimia y metáfora en opinión de Varela, porque su definición era estricta en tanto que referido a sistemas vivos en su expresión mínima 0 celular. Nosotros diríamos que lo hizo como «modelo conceptual» y como tal fue aplicado a otros campos; Luhman emplea el término en sus estudios sobre el sistema social (1998) y en sus referencias a los sistemas culturales (1990, 1996). También Dupuy (1989) hace lo propio, aplicando el concepto al estudio de los sistemas sociales; el propio Varela aceptó que con notable precisión. Nosotros hemos tomado el término como elemento radical del concepto de autonomía y hemos definido su propiedad funcional para todo proceso de cambio derivado de la situación de incorporación cultural (García Carrasco y García del Dujo, 2001): en cualquier proceso de influencia exterior, las consecuencias en el interior no podrán explicarse a partir de las propiedades del foco de influencia, sino que la comprensión implica la identificación de los mecanismos y de los estados de cosas en el interior. Se trata de un proceso causal, cuya explicación requiere de los conceptos de autonomía y auto referencia.

Este ejemplo da muestra de las dificultades que entraña la construcción de una teoría de la educación que incorpore la perspectiva biológica, aunque a todas luces se muestre en la actualidad como necesaria. La necesidad de su incorporación arranca de la necesaria revisión de la imagen lockiana de la tabla rasa. La formación, hoy, debe ser también comprendida como exigencia vital, biológicamente condicionada y vitalmente comprendida. Algunos reducen esta

(1) Son muchos los autores que en este momento practican esta relectura del mundo de la vida sin contraponer la historia evolutiva a una antropología exclusiva. Un ejemplo de interpretación general puede entenderse el libro de Dennett (2000), donde la pauta que conecta es el concepto de intencionalidad. Una propuesta de diversos itinerarios puede verse en Capra (1998). 
cuestión al mero problema de la genética del comportamiento o al carácter hereditario de aptitudes y disposiciones, lo que convierte el problema general en un catálogo de curiosidades. Las relaciones entre biología y comportamiento se extienden más allá de la cuestión de la heredabilidad de los caracteres, bajo la cual subyace de nuevo el dualismo segregador naturaleza-cultura. Incide directamente en la construcción de una antropología que muestre «el conocimiento como emergencia encarnada, la conciencia en tanto experiencia vivida y realización biológica en un todo indisoluble» (Varela, 2002, 13).

Y, construida la perspectiva, el paso siguiente es la diferenciación de organizadores temáticos que desde ella permitan aportar elementos a la teoría de la educación para la construcción de una matriz disciplinar. Su incardinación podría estar tanto bajo el palio de la biología de la educación como del de la antropología de la educación. La opción por uno u otro título no es la cuestión fundamental; lo que parece menos discutible es que han de ser organizadores de capítulos de Teoría de la Educación. El riesgo está en entender, reductivamente, que el proyecto de matriz disciplinar se reduce a «introducir» en el conocimiento biológico, cuando el objetivo representa más bien un programa de investigación fascinante, abierto a la cooperación entre grupos de trabajo, en un amplio horizonte temporal.

La comodidad reflexiva ha llevado en ocasiones a plantear las cosas de otra manera: si el proceso formativo se enmarca en un contexto de incorporación cultural, los organizadores temáticos básicos estarán relacionados con la tradición académica de la antropología cultural; el tema más propio para el campo de conocimiento de la educación sería el de la antropología de la transmisión de cultura y sus problemas relacionados, entre otros, la relación entre culturas. Entendemos, sin embargo, que este modo de reflexionar es el que ha ido alejando la reflexión educativa de las cuestiones fundamentales de la Biología. Ha predominado justificadamente la construcción de la teoría de la educación desde lo que denominaríamos sociofilia; creemos importante reclamar la aportación que proporciona a la comprensión la actitud denominada biofilia (Wilson, 1989). 
En este sentido encontramos un paquete de temas fundamentales para la comprensión de la formación como necesidad vital de los humanos y de las relaciones básicas entre cultura y vida. Señalaremos, a título meramente indicativo, tres estrechamente relacionados.

El primero ya lo hemos aludido: el propio fenómeno de la vida analizado en tanto que acontecimiento en el que evoluciona la autonomía, la identidad, el conocimiento y la valoración vital de los sucesos del mundo; el mundo de la vida como marco de referencia del significado y del sentido.

Un segundo tema fundamental, tanto en biología como dentro del campo de la formación, viene señalado por el concepto de ambiente; sus definiciones dominantes obligan a la reconstrucción de su significado. Habitualmente se toma como realidad que envuelve, zona y nicho de ocupación 0 entorno de exigencias a las que el organismo ha de adaptarse 0 perecer; este concepto de ambiente ha de reconstruirlo la Teoría de la Educación, para hacer coherente su estudio con el marco general en el que se entiende el fenómeno de la vida y el fenómeno integral de la educación. Como afirma Lewontin (2000) «ha llegado el momento de reconsiderar la relación entre interno y externo, entre organismo y ambiente». En este punto se proponen los conceptos de construcción e interacción como herramientas conceptuales básicas; el entorno que corresponde a la identidad orgánica está formado por las condiciones relevantes para la dinámica de sus funciones autónomas (Vendrell, Guerrero y Berlanga, 2004). Desde este punto de vista son los propios organismos los que instituyen activamente su mundo; su quehacer en el medio modula las condiciones locales. De nuevo la historia de la vida puede ser narrada como una evolución en coderiva de las relaciones entre organismo y ambiente (Margulis y Olendzenski, 1996). La biosfera no está meramente formada por el catálogo de los seres vivos, sino también por el ambiente en el que vivimos y que la propia evolución contribuyó a construir. Tal vez éste sea el marco adecuado en el que situar la formación de la sensibilidad ambiental, regida por el principio de responsabilidad, cuando el organismo considerado tiene como profesión vital la cultura. Una 
parte relevante de la creación cultural humana consiste en su contribución ambiental, para bien o para mal: H. Joñas ha puesto de relieve la importancia que este planteamiento tiene para la deliberación sobre el principio de responsabilidad.

El tercer tema relevante para la comprensión de la formación, que también se ha ido alejando de los discursos pedagógicos, es el pasado de la especie. De nufvo señalamos que la perspectiva no es la de la mera introducción a la Paleoantropología, sino la respuesta rigurosa a interrogantes vigorosos que se construyen desde y para la comprensión de la formación. El interés debiera estar dirigido a profundizar en la bioestructura del comportamiento mediante el procedimiento de retroceder y dejar que afloren los caracteres antropológicos fundamentales (Arsuaga y Martínez, 1998). Son abundantes los ejemplos para este ejercicio, con los que aprender a trabajar y contribuir a la matriz disciplinar. El organizador temático podría expresarse como evolución de la conciencia (Orstein, 1994) o como evolución de la mente (Mithen, 1998): ¿cómo somos los humanos para necesitar la cultura para vivir? (Carrithers, 1995), ihasta dónde es importante la diversidad biológica aparente entre tipos humanos (Lewontin, 1984).

La parte que corresponde a la Teoría de la Educación consiste en mostrar cómo la historia evolutiva de la especie contribuye a la comprensión del modo de ser y del modo de actuar humano. Por ejemplo, ha dominado una antropología que situaba al Homo faber y a la herramienta en un lugar de privilegio; sin embargo, todo indica que a un nivel tanto 0 más importante estuvo la cooperación social (Picq y Coppens, 2002) implicando en ello incluso la evolución de la mano (Alba, Moyá y Koler, 1995) perspectiva esta que se refuerza incluso desde una antropología comparada con las culturas de los grandes simios (Waal, 2002). Son muchos los temas particulares desde los que ir construyendo esa perspectiva que rompa la segregación e integre la naturaleza y la cultura (García Carrasco, 2002).

Toda esta contribución aboca a la necesidad de una revisión profunda de la perspectiva oculta desde la que el significado, el sentido y el proceso de 
la formación se obtienen con exclusividad desde el ámbito de la cultura; en el fondo es la perspectiva lockiana de la tabla rasa, a cuya obligada revisión se invita desde los más diferentes campos disciplinares. Entendemos que esa revisión es la clave para el trabajo futuro de construcción de la Teoría de la Educación que, desde la referencia al mundo de la vida, estamos proponiendo (Pinker, 2003).

\section{Relectura de la (teoría de la) educación desde la tecnología}

Esta segunda perspectiva, que también reclama el renovado esfuerzo de integración en la Teoría de la Educación, se justifica por un doble motivo. En primer lugar, por el hecho de que estamos asistiendo a una auténtica contingencia cultural (Laszlo, 1997), habitualmente denominada Sociedad de la Información (Castells, 1996, 1997 y 1998) en cuya raíz se encuentra una profunda transformación tecnológica. Tal vez se podrían resumir los grandes ejes que señalan la contingencia cultural diciendo que asistimos a una revolución de las comunicaciones, una profunda transformación de las organizaciones y una transcendental conmoción en el acceso al mundo de la vida, del cual es indicio el denominado Proyecto Genoma Humano (Echeverría, 2003). El segundo motivo se deriva del hecho de que en los últimos treinta años de investigación pedagógica se ha venido contraponiendo el denominado modelo tecnológico al llamado histórico social, perfilado este último con diferentes matices. La contraposición se producía porque, en ocasiones, la concepción de la acción pedagógica como acción técnica tenía como telón de fondo una filosofía de la tecnología claramente insuficiente, que marginaba en el discurso el contexto sociocultural en el que adquirían sentido las políticas educacionales. Pero la discusión y el planteamiento de perspectivas pluridisciplinares enriquecieron de manera innegable el 
panorama teórico disponible. El problema que resta es, de nuevo, el de la integración: conseguir un marco integrado para el funcionamiento de la mente en la cultura, lo que en el fondo no es otra cosa que ampliar la comprensión de la formación y mejorar la comprensión de la tecnología.

En este caso son varios los supuestos de los que partir. El primero, recuperar la perspectiva biofílica anteriormente descrita; el segundo, superar en la reflexión la orientación individualista de un sujeto aislado en sus relaciones mentales con el mundo, como venía siendo ideológicamente reforzado desde la Ilustración. En definitiva, integrar el estudio de los procesos mentales y los escenarios histórico-sociales en los que transcurren e incorporar en la reflexión el hecho de que las prácticas culturales expresan y transforman los procesos mentales superiores, asumido todo ello por la perspectiva denominada psicología cultural (Colé, 1999). En este sentido, la unidad de análisis no será el proceso mental sino su inserción como elemento en una estructura de acción (Leontiev, 1984) y la acción en un contexto de práctica intersubjetiva (Wenger, 2001), viendo las funciones mentales emergiendo de ella y considerando la formación como acción mediada (Wertsch, 1993).

Esta perspectiva reconoce en Vygotski (1996) uno de los autores que trazaron las orientaciones principales de la perspectiva. Frecuentemente se identifica este programa de investigación con la expresión sociocultural; sin embargo, en su raíz era más bien sociohistórica, pues pretendía integrar la relevancia de los movimientos sociales y el valor explicativo de la dinámica histórica. En el concepto de historia no solamente se recogía la importancia de la historia cultural, sino también la de la historia evolutiva, ontogenética y filogenético, incluyendo así en el núcleo del programa la perspectiva biológica. De todo ello da prueba la obra fundamental del autor Pensamiento y lenguaje (1982) y su referencia a lo que denominaba dominios genéticos; en ellos se tomaba en consideración incluso el conocimiento etológico accesible a través de las investigaciones de Kohler con simios. Sin embarg0, el desarrollo de la biología de la mente fue favorecido más por Luria y 
la implicación de procesos históricos-sociales en la configuración de las prácticas formativas fue desarrollada por otros muchos autores.

La acción mediada fue especialmente estudiada por la corriente central vygotskiana en relación con instrumentos mediadores y de manera especial el lenguaje, en cuanto instrumento de los instrumentos de comunicación, y las herramientas, en tanto que mediadores de la mente aplicada a la acción; por eso, unos y otros, fueron considerados instrumentos psicológicos (Kozulin, 2000). Sin embargo, el desarrollo fundamental de la teoría de la mediación instrumental siguió la línea de la mediación del signo y de la mediación del lenguaje, estudios en los que Bajtin fue una autoridad todavía reconocida (Silvestri y Blanck 1993; Vauthier y Cátedra, 2003). Fue menos atendida la teoría de la mediación de otros instrumentos; para la Teoría de la Educación quedan muchas líneas abiertas en la investigación de la acción mediada por la tecnología.

Ante todo, superar un sesgo que debilita las posibilidades teóricas y las motivaciones prácticas; el sesgo consiste en tomar como atractor de la reflexión al artefacto, a la herramienta, a la máquina. En la perspectiva del artefacto el protagonista es la máquina y el modelo causal es el esquema lineal que se vislumbra desde ella. En el inicio, el sesgo llevaba aparejado, especialmente en la década de los setenta y ochenta del pasado siglo, una más que notable connivencia con el conductismo y el empleo de la cibernética como marco para una gestión mecano-técnica del aprendizaje; una suplantación del concepto de autonomía por el de control. Las claves fueron la enseñanza programada (Fry, 1966) y la pedagogía por objetivos, lo que llevó a una crítica dura de ese modelo tecnológico, junto a la contraposición de una pedagogía de orientación sociohistórica con mayor énfasis en la pedagogía crítica que en el desarrollo del programa descrito de la acción mediada (Gimeno, 1988). Una propuesta de mayor calado fue tomar el aparato conceptual de la cibernética para el estudio del proceso de formación, entendido como sistema complejo adaptativo, de lo que dio testimonio la obra de H. Frank (1976) y el meritorio trabajo de A. Sanvisens 
(1984). La denominada escuela de Palo Alto hizo aportaciones importantes a una cibernética con sujeto, también denominada segunda cibernética, en la que destacó, entre otros, H. Foerster (1996).

Vygotski propuso un marco más amplio para la mediación instrumental, aunque centrando la investigación especialmente en el lenguaje y el signo. Evidentemente, este punto de vista requiere importantes modificaciones en la propia filosofía de la tecnología, en nuestra opinión sesgada hacia las repercusiones sociales de los desarrollos tecnológicos y centrados estos en los artefactos (García Carrasco, 1993, 1996). Algunos autores han planteado un criterio más general para el concepto de tecnología: «conocimientos de base científica que permiten describir, explicar, diseñar y aplicar soluciones técnicas a problemas prácticos de forma sistemática y racional» (Aibar y Quintanilla, 2002, 16). En las prácticas formativas, la comprensión del problema práctico que en ellas aflora subraya el contexto de probabilidad e incertidumbre, como corresponde a sistemas muy complejos, e incita a la deliberación sobre estrategias y tácticas, dentro de las cuales adquieren sentido las mediaciones instrumentales. Son los actores quienes gestionan los instrumentos dentro de los propósitos de la acción, en la zona de construcción del conocimiento; de ahí que los instrumentos hayan de reflexionarse desde la virtualidad que los actores descubren en ellos, dentro de la zona intersubjetiva en la que se promueven las prácticas de formación.

Todo ello induce a que la Teoría de la Educación debe investigar la tecnología desde el punto de vista del sistema técnico, que en principio, es un «dispositivo complejo compuesto de entidades físicas y de agentes humanos, cuya función es transformar, de forma eficiente, algún tipo de cosas para obtener determinados resultados característicos del sistema» (Idem, 21); aun así, el sistema técnico se analiza desde la perspectiva que tiene como modelo la actividad en el sistema productivo. En cualquier caso, en la perspectiva del sistema técnico, adquiere importancia de primer rango la calidad de la alfabetización tecnológica de los agentes intencionales que operan con los artefactos y la de los planes en vistas a metas valoradas, 
lo que permitiría recuperar, para la mediación tecnológica en la acción formativa, el papel indiscutible de la praxis reflexiva, reorientándose así, la teoría hacia la perspectiva vygotskiana. En este contexto se impone la deliberación sobre la mejor aplicación de los recursos instrumentales, siempre machihembrados a la acción humana (Linz, 2000), y la deliberación sobre las metas consensuadas para la acción compartida.

La operación mental superior brota de una experiencia intersubjetiva dentro de un sistema de acción mediado por instrumentos. La meta no está en la presencia del instrumento, equipamiento técnico, sino en la conformación de experiencia. La pedagogía, en cuanto forma de investigación y deliberación sobre la práctica formativa, conlleva extraer, imaginar y probar el patrón de actividad, que desborda el ámbito de la herramienta y le imbuye del ejercicio creativo de la razón humana, le inyecta razón práctica. Desde este punto de vista, la misma cultura podría expresarse como modos cooperativos de participar en las técnicas, modos sociales de asumirlas, de colaborar en ellas e incorporarlas a los patrones de actividad de quienes las aprenden, en vistas al proyecto colectivo de calidad de vida, que la comunidad responsablemente valora. Están implicadas en la tarea de formación, tanto la lógica del diseño del instrumento como la lógica del uso del instrumento en la tarea compartida del plan de formación; esto siempre ha sido así, en todas las épocas (García Carrasco, 2005).

En este sentido, la tecnología informacional abre varias líneas en la investigación. La primera, que podíamos calificar de básica, se suma a la investigación sobre el propio funcionamiento de la mente, en la medida que se toma el ordenador como instrumento para la simulación y la emulación de operaciones (Jonson-Laird, 2000), especialmente para estudiar sus posibilidades y límites, dado que en ese contexto se parte del criterio, en parte metafórico y en parte real, de que la investigación opera con «objetos con mente» (Riviere, 1998), lo que, a su vez, plantea una confrontación teórica general respecto a lo ya señalado en el epígrafe de la investigación biológica. Se abre así una puerta de reflexión que involucra, entre otros, el 
ámbito de las operaciones cognitivas así investigadas (Hawkins y Blakeslee, 2005) y las implicaciones teóricas de la introducción de creencias y deseos, de comprensión de situaciones, intenciones... etc.

Todo ello nos compromete, a su vez, con la deliberación de la virtualidad y los límites de la propia teoría y tecnología de la información para la comprensión de la acción mediada en la zona de construcción del sujeto, el ámbito propio de las acciones de formación (Caffarel Serra, 1996). Queda implicada, pues, la Teoría de la Educación en el análisis y valoración de la sociedad de la información y de toda la literatura de acompañamiento, desde la filosofía de la actividad humana hasta la teoría inducida a partir de la construcción de simuladores de espacios de acción formativa, pasando por la reflexión práctica de acciones formativas mediadas por la tecnología. Precisamente, el debate en el que están implicadas las ciencias naturales y humanas está potenciando la inversión de la imagen: de un ordenador metáfora para un cerebro se pasa a un cerebro metáfora para un ordenador.

El rechazo en el ámbito de la reflexión pedagógica del denominado modelo tecnológico estribaba en que la definición de técnica que asumía el modelo era la de un algoritmo de acción causalmente vinculado, de manera lineal y eficiente, a un patrón de comportamiento. Esto no era proyectar una acción técnica sino buscar, como siempre, la receta. Lo que muchos denominan reflexión sobre la práctica de formación es precisamente una demanda de filosofía sobre las acciones mediadas por instrumentos: es deliberación sobre la práctica-con las técnicas y no sólo reflexión sobre la tecnología. La novedad está en que la brecha que separa la compleja tecnología de los agentes de la formación obliga a plantear las líneas de investigación como necesariamente interdisciplinares.

Y con base en este punto de vista de la acción mediada de formación inserta en un sistema técnico se abren otros capítulos a la investigación teórica: el correspondiente a la deliberación sobre los componentes artificiales, crítica de los existentes y diseños posibles; la reflexión sobre los agentes 
intencionales implicados en las prácticas, dentro de los que se podrían señalar cuatro tipos: investigadores de ciencia y tecnología, ingenieros productores de hardware, ingenieros diseñadores de software, gestores del sistema (administradores, supervisores, grupos interdisciplinares de apoyo y producción de contenido, e-moderadores, e-tutores) y usuarios (cibernautas, telepolitas, piratas, delincuentes, formadores), lo que lleva a la necesidad de ampliar el concepto de plan de formación a la idea de proyecto cooperativo, sin olvidar la deliberación sobre la estructura y el patrón de interacciones entre los componentes del sistema técnico, desde el punto de vista de su significación cultural, lo que viene a replantear la cuestión de los intereses generales y metas de los espacios de formación construidos, la reconfiguración de las prácticas formativas en el nuevo espacio de actividad creado por la contingencia tecnológica, la deliberación sobre las responsabilidades de gestión y de supervisión del espacio formativo promovido, et. En todo ello es tanto más importante y urgente la deliberación teórica cuanto limitada es la experiencia acumulada, pues puede originar, antes incluso que la experiencia de fracaso, la decepción de los actores.

\section{Recuperando y abriendo per spectivas}

En nuestra investigación de los últimos años estamos suponiendo y comprobando que el perfil que presenta en la actualidad la condición tecnológica del ser humano se comprende mejor en cuanto fenómeno cultural que meramente tecnológico y, por tanto, responde mejor a la concepción de cultura en sentido primario que en cuanto creación o patrimonio cultural; esto segundo lo damos por supuesto, pero lo consideramos insuficiente para los intereses de nuestra disciplina.

No negamos que las nuevas tecnologías sean herramientas o instrumentos pertenecientes a una fase ulterior del desarrollo económico y social del 
ser humano y que en este sentido puedan y deban ser usufructuadas en educación, pero pensamos que estas tecnologías

«no son, ni deben ser consideradas por más tiempo simples artefactos 0 instrumentos que posibilitan la acción 0 manipulación del entorno por el sujeto. Las nuevas tecnologías son algo más que el elemento o componente material, instrumental, siempre presente en los contextos tradicionales de educación; no negamos que lo sigan siendo, pero decimos que son algo más que eso. En este sentido no son meros artefactos, sino artificios capaces de generar nuevos contextos, escenarios diferentes. Son las dos caras, al menos, que presentan las nuevas tecnologías: instrumentos, materiales, que generan estructuras que posibilitan acciones y viceversa. Y sobre esta doble perspectiva pivota la mayor potencialidad y responsabilidad que tienen las nuevas tecnologías, que no es otra que la posibilidad de incidir sobre los marcos de pensamiento, acción y representación del sujeto (García del Dujo, 2005a, p. 17).

En estos momentos, ya no quedan integrados y apocalípticos; unos y otros han terminado por aceptar que las nuevas tecnologías son ya herramientas imprescindibles en cada vez más ámbitos de la realidad y, lo que es más importante para nosotros, han empezado a pensar que los cambios que se están produciendo en las formas de acción de los más diversos campos de la realidad no deben interpretarse únicamente en términos de correspondencia lineal y automática con la introducción y presencia de esas tecnología sino que requieren una lectura más global y, sobre todo, dialéctica y social de su comportamiento y uso en contextos específicos.

Esto significa una reorientación, metodológica y conceptual, importante, pues, al poner el énfasis, más que en la tecnología en sí misma, en sus usos y representaciones, se está dando un paso notable para el alcance y desarrollo de esas tecnologías: de ser consideradas como meras herramientas 0 instrumentos de acción y producción, instrumentos culturales, por supuesto, pero al fin y al cabo simples objetos 0 artefactos con una función meramente instrumental, comienzan a ser vistas como artefactos con potencialidad, por sus características técnicas, para generar no sólo escenarios de pensamiento sino también de acción e interacción, lo que termina por conducir a una interpretación de las nuevas tecnologías como cultura en cuanto que son capaces de generar sitios o lugares donde la gente puede hacer cosas, 
moverse, expresar y compartir opiniones, sentimientos, valores... manipular e intercambiar objetos... y crear así progresivamente comunidades. Las nuevas tecnologías, a través de los entornos que generan, están visualizando lo mejor de su naturaleza y comportamiento como lugares donde se construye y reconstruye la cultura (Benedikt, 1991; Hiñe, 2001; García del Dujo, 2005b).

Este salto en la visión e interpretación de las nuevas tecnologías es importantísimo para la educación, pues desde ese mismo momento, no antes, es probable que se empiece a pensar, a aceptar y a actuar en ellas como si de «otros escenarios reales» se tratase y, en consecuencia, se les reconozca con rigor plena capacidad formativa, la misma que se atribuye a los contextos tradicionales, en cuanto que allí puede llevarse a cabo una serie de actividades que «tipifican» el proceso educativo, aunque no de la misma manera. Bien puede decirse que estamos atravesando por un momento crítico en el uso de las nuevas tecnologías (García del Dujo, 2003), que se corresponde, a su vez, con formas particulares de pensarlas, interpretarlas, verlas e imaginarlas: momento crítico, pues, también para la reflexión que en este sentido le corresponde hacer a la Teoría de la Educación.

Necesitamos una pedagogía que de cuenta, que explique satisfactoriamente los resultados formativos obtenidos por unos sujetos cuyo comportamiento está mediado tecnológicamente y tiene lugar en entornos virtuales y, a su vez, necesitamos una tecnología que posibilite el tipo de acciones requeridas por esa interpretación pedagógica de los procesos formativos; lo segundo no es problema, lo primero no parece preocupar demasiado (García del Dujo, 2005c; Touriñan López, 2005). Pedagogía y tecnología quedan implicadas mutuamente en nuestra visión, siendo aquella algo más que un trazado de estrategias y espacios diseñados y esta mucho más que un conjunto de herramientas.

$Y$ en nuestra investigación de los últimos años estamos también entendiendo que una Teoría de la Educación que permita avanzar en el conocimiento de aquello que acontece en la relación pedagógica ha de abrirse necesariamente a la pluridimensionalidad del ser humano, a su desmesurada complejidad, a su indisociable realidad de ser viviente y sujeto cultural. 
Esta Teoría de la Educación ha de tratar con especial finura todo cuanto concierne no ya al cómo aprendemos, regulamos nuestros afectos 0 enseñamos a comportarnos debidamente, sino más bien a lo que se entiende por conocer, emocionarnos o desarrollarnos como seres éticos. Porque, una vez comprendido esto, una vez elaborada una buena teoría al respecto, el quehacer del educador se verá sensiblemente facilitado y mejorada su posibilidad de concebir estrategias pedagógicas lo suficientemente flexibles como para adaptarse a las necesidades de cada sujeto.

Ahora bien, en todos estos empeños nos encontraremos inevitablemente con el modo de operar de un ser vivo que asimila cultura, que se muestra sensible a su entorno, físico y social, pero que también manifiesta sus límites e inercias a las influencias ambientales. Un ser vivo que se autodetermina, aun siendo educable, y que, pese a ello, está asimismo biológicamente equipado para hacerse responsable de sus conductas al tomar conciencia de sus consecuencias.

Si nos entrenamos para pensar la condición humana como un cuerpo capaz de producir un mundo virtual y de habitar en él, experimentándolo realmente, entonces el cuerpo, el entorno y el artificio serán concebidos como un conjunto funcional (Cyrulnik, 2002, 21).

Para reflexionar sobre los problemas relacionados con el modo como los seres humanos conocen, sienten y se relacionan, al pedagogo no le quedará otro camino en el futuro que familiarizarse con ciertos conocimientos aportados por algunas disciplinas biológicas, especialmente la Neurobiología y la Etología, porque sólo entonces estará en condiciones de valorar con criterio contrastable en qué medida pueden ser mejoradas las prácticas educativas y la propia formación de profesores y educadores. Una Teoría de la Educación alejada de esos conocimientos se ve privada del soporte empírico que otorga una cierta fundamentación a sus planteamientos epistemológicos, a aquello que entendemos por «realidad», «verdad» 0 «explicación», así como a las posibles maneras de entender el aprendizaje humano e interpretar las múltiples formas de desadaptación que se manifiestan en las relaciones educativas. 
Intentar comprender la naturaleza de los procesos cognitivos al margen de la estructura y organización del cerebro, de su historia evolutiva y de los intereses que la han impulsado o de la dinámica cerebro/mente surgida de la propia actividad cerebral, no parece hoy la postura más razonable. Para la mayoría de los biólogos, ni el cerebro consiste en una especie de ordenador, aunque algunas de sus funciones puedan ser simuladas con él, ni la mente algo que pueda considerarse independiente de la dinámica cerebral.

Los procesos comunicativos, esenciales en el ámbito de las relaciones educativas, tampoco pueden hoy ser cabalmente interpretados sin los aportes de la segunda cibernética y la consideración del cerebro como un sistema autoorganizado que responde a las influencias ambientales de manera no determinable, dado el carácter histórico de las estructuras que lo conforman. Cualquier conducta puede ser entendida entonces como una comunicación si modifica en algún sentido dichas estructuras del sujeto receptor, bien sea para inducirle una respuesta emocional, motora o cognitiva. De esta manera,

se lo proponga 0 no, el docente participa, con su estar, hacer y decir, en la dinámica general del sistema «aula» y no sólo en el pretendido trasvase de unos contenidos disciplinares inefablemente vehiculados a través del lenguaje (Asensio, 2004, 151).

De ahí la necesidad de acercar la Teoría de la Educación a la denominada «pragmática de la comunicación» y poder así interpretar la naturaleza holística de los procesos comunicativos en los seres humanos.

Éstos son los pilares sobre los que estamos haciendo y pensando la Teoría de la Educación; ello no significa para nosotros reduccionismos ni determinismos de ningún tipo, ni biologistas ni tecnológicos, ni individuales ni culturales. Al contrario, entendemos que tomar la formación del ser humano como un proceso de acción mediada, dentro de una comunidad de prácticas culturales, tiene potencial suficiente para integrar la deliberación sobre las virtualidades tecnológicas que lleva consigo la actual contingencia cultural y para colmar progresivamente la brecha semántica que viene separando no sólo las ciencias naturales y las ciencias sociales sino también la Teoría de la Educación de otras disciplinas próximas e imprescindibles. 
Aibar, E. y Quintanilla, M. A. (2002). Cultura tecnológica. Estudios de ciencia, tecnología y sociedad. Barcelona: Horsori Editorial.

Alba, D. M.; Moyá, S. y Köhler, M. (1995). El origen de la mano humana. Investigación y ciencia, febrer0, 46 y ss.

Arsuaga, J. L. y Martínez, I. (1998). La especie elegida. La larga marcha de la evolución humana. Madrid: Temas de hoy.

Asensio, J. M. (1997). Biología y educación. Barcelona: Ariel.

Asensio, J. M. (2004). Una educación para el diálogo. Barcelona: Paidós.

Benedikt, M (ed.) 1991. Cyberspace: First Steps. Cambridge: MIT Press.

Briggs, A. y Burke, P. (2002). De Gutenberg a Internet. Una historia social de los medios de comunicación. Madrid: Taurus.

Broncano, F. (ed.) (1995). Nuevas meditaciones sobre la técnica. Madrid: Trotta.

Caffarel Serra, C. (comp.) (1996). El concepto de información en las Ciencias Naturales y Sociales. Madrid: Universidad Complutense.

Capra, F. (1998). La trama de la vida. Una nueva perspectiva de los sistemas vivos. Madrid: Anagrama.

Carrithers, M. (1995). ¿Por qué los humanos tenemos culturas? Madrid: Alianza.

Castells, M. (1996, 1997, 1998). La era déla información. Volumen I, II y III. Madrid: Alianza.

Changeux, J. P. y Ricoeur, P. (1999). Lo que nos hace pensar. La naturaleza y la regla. Barcelona: Península.

Cole, M. (1999). Psicología cultural: una disciplina del pasado y del futuro. Madrid: Morata.

Cyrulnik, B. (2002). El encantamiento del mundo. Barcelona: Gedisa.

Davies, K. (2001). La conquista del genoma humano. Craig Venter, James Watson y la historia del mayor descubrimiento científico de nuestra época. Barcelona: Paidós.

Delbrück, M (1989). Mente y materia. Madrid: Alianza.

Dennett, D. C. (2000). Tipos de mentes. Hacia una comprensión de la conciencia. Barcelona: Debate.

Dupuy, J. P. (1989). Ordres et désordres. París: Seuil.

Echeverría, J. (2003). La revolución tecnocientífica. Madrid: Fondo de Cultura Económica.

Foerster, H. von (1996). Las semillas de la cibernética. Obras escogidas. Barcelona: Gedisa.

Frank, H. G. y Meder, B. S. (1970). Introducción a la pedagogía cibernética. Buenos Aires: Troquel.

Fry, E. B. (1966). Máquinas de enseñar y enseñanza programada. Magisterio Español, Madrid.

García Carrasco, J. (1993). Técnica, Tecnología y educación. Análisis y revisión de conceptos. Arbor, 145, pp. 121-157.

García Carrasco, J. (1996). Acción técnica y acción pedagógica. En Tejedor, F. J. y García Valcárcel, A. Perspectivas de las nuevas tecnologías en la educacion. Madrid: Narcea, pp. 37-63. 
García Carrasco, J. (2002). Reconocimiento e interioridad. Tramas que conectan el mundo de la vida. En Hernández, J. M.; Lecuona, M. del P. y Vega, L. (eds.) La educación y el medio ambiente natural y humano. Salamanca: Ediciones Universidad de Salamanca, pp. $139-167$.

García Carrasco, J. (2004). La comprensión de la vivienda como un dominio vital de los seres humanos. Revista Española de Pedagogía, 228, pp. 229-256.

García Carrasco, J. (2005). Virtualidad formativa de espacios mediados por la tecnología. En Peiró i Gregori, S. Nuevos espacios y nuevos entornos de educación. Alicante: Editorial Club Universitario.

García Carrasco, J. y García del Dujo, A. (1997). Presupuestos de la cultura como acontecimiento antropológico. Teoría de la educación. Revista Interuniversitária, 9, pp. $21-40$.

García Carrasco, J. y García del Dujo, A. (2001). Teoría de la educación. Volumen II. Procesos primarios de formación del pensamiento y la acción. Salamanca: Ediciones Universidad de Salamanca.

García del Dujo, A. (2003). Momentos críticos en el uso de las nuevas tecnologías en educación, en IX Congreso Interuniversitário de Teoría de la Educación. Calidad, equidad y educación. San Sebastiân.

García del Dujo, A. (2005a). Formación permanente y nuevas tecnologías. En García del Dujo, A.; Martín García, A. V. y Pérez Grande, M. D. Procesos defromación on Une. Salamanca; Amarú Ediciones, pp. 15-37.

García del Dujo, A. (2005b). Pedagogia degli ambienti virtuali di apprendimento, Pedagogía e Vita, 63 (2), pp. 62-82.

García del Dujo, A. (2005a). Procesos de formación en entornos virtuales. En García del Dujo, A.; Martín García, A. V. y Pérez Grande, M. D. Procesos defromación on Une. Salamanca; Amarú Ediciones, pp. 39-68.

García del Dujo, A.; Martín García, A. V. y Pérez Grande, M. D. (2005). Procesos de fromación on Une. Salamanca: Amarú Ediciones.

Gimeno Sacristán, J. (1988). Pedagogía por objetivos: obsesión por la eficiencia. Madrid: Morata.

Hawkins, J. y Blakeslee, S. (2005). Sobre la inteligencia. Madrid: Espasa.

Hiñe, Ch. (2001). Virtual Etnography. London: Sage.

Jonas, H. (1997). Técnica, medicina y ética. La práctica del principio de responsabilidad. Barcelona: Paidós.

Jonas, H. (1995). El principio de responsabilidad. Ensayo de una ética para la civilización tecnológica. Barcelona: Herder.

Jonson-Laird, P.H. (2000). El ordenador y la mente. Barcelona: Paidós.

Kozulin, A. (2000). Instrumentos psicológicos. La educación desde una perspectiva sociocultural. Barcelona: Paidós.

Jonas, H. (2000). El principio vida. Hacia una biología filosófica. Valladolid: Trotta.

Laszlo, E. (1997). La gran bifurcación. Barcelona: Gedisa.

Leontiev, A. N. (1984). Actividad, conciencia y personalidad. México: Editorial Cartago.

Lewontin, R. C. (1984). La diversidad humana. Barcelona: Labor.

Lewontin, R. C. (2000). Genes, organismo y ambiente. Barcelona: Gedisa.

Linz, M. (1995). Conocer y actuar a través de la tecnología. En Broncano, F. (ed.) Nuevas meditaciones sobre la técnica. Madrid: Trotta.

Luhman, N. (1990). Sociedad y sistema: la ambición de la teoría. Barcelona: Paidós.

Luhman, N. (1996). Teoría de la Sociedad y Pedagogía. Barcelona: Paidós. 
Luhman, N. (1998). Sistemas sociales: lineamientos para una teoría general Barcelona: Anthropos.

Margulis, L. y Olendzenski, L. (eds.) (1996). Evolución ambiental Madrid: Alianza.

Margulis, L. y Sagan, D. (1996). ¿Qué es la vida? Barcelona: Metatemas-La Caixa.

Margulis, L. y Sagan, D. (2001). Microcosmos. Cuatro mil millones de arios de evolución desde nuestros ancestros microbianos. Barcelona: Metatemas-La Caixa.

Maturana, H. y Varela, F. (1984). El árbol del conocimiento. Las bases biológicas del entendimiento humano. Santiago de Chile: Editorial Universitaria.

Maturana, H. y Varela, F. (1990) El árbol del conocimiento. Las bases biológicas del conocimiento humano. Madrid: Debate.

Maynard-Smith, J. y Szathmáry, E. (2001). Ocho hitos de la evolución. Del origen de la vida a la aparición del lenguaje. Barcelona: Metatemas-La Caixa.

Mithen, S. (1998). Arqueología de la mente. Orígenes del arte, de la religión y de la ciencia. Barcelona: Grijalbo-Mondadori.

Orstein, R. (1994) La evolución de la conciencia. Barcelona: Emecé Editores.

Piaget, J. (1977) Biología y conocimiento: ensayo sobre las relaciones entre las regulaciones orgánicas y los procesos cognoscitivos. Madrid: Siglo XXI.

Picq, P, y Coppens, Y. (2002). Le propre de l'homme. En Aux Origines de l'humanité, vol II. París: Fayard.

Pinker, S. (2003). La tabla rasa. La negación moderna de la naturaleza humana. Barcelona: Paidós.

Rivière, A. (1998). Objetos con mente. Madrid: Alianza.

Sanvisens i Marfull, A. (1984). Cibernética de lo humano. Barcelona: Oikos-Tau.

Scheler, M. (1979). El puesto del hombre en el Cosmos. Buenos Aires: Losada.

Silvestri, A. y Blanck, G. (1993). Bajtin y Vigotski: la organización semiótica de la conciencia. Barcelona: Anthropos.

Smith, C. U. M. (1977). El problema de la vida. Ensayo sobre los orígenes del pensamiento biológico. Madrid: Alianza.

Varela,F. (1996). De máquinas y seres vivos. Santiago de Chile: Editorial Universitaria.

Varela, F. (1989). Autonomie et connaissance. Essai sur le vivant. Paris: Seuil.

Varela, F. (2002). El fenómeno de la vida. Santiago de Chile: Dolmen ediciones.

Vendrell, B.; Guerrero, R. y Berlanga, M. (2004). Ecosistemas mínimos. Investigación y ciencia, enero, pp. 36-37.

Vygotski, L. S. (1982) Pensamiento y lenguaje. Obras escogidas T. II. Madrid: Visor.

Vygotski, L. S. (1996) El desarrollo de los procesos psicológicos superiores. Barcelona: CríticaMondadori.

Waal, F. (2002). El simio y el aprendiz de Sushi. Reflexiones de un primatólogo sobre la cultura. Barcelona: Paidós.

Waltz, S. B. (2002). Giving Artefacts a Voice? Bringing into Account Technology in Educational Analysis. Educational Theory, 54, 2, pp. 157-172.

Vauthier, B. y Cátedra, P. M. (2003). Mijail Bajtin en la encrucijada de la hermenéutica y las ciencias humanas. Salamanca: Sociedad de Estudios Medievales y Renacentistas.

Wenger, E. (2001). Comunidades de práctica. Aprendizaje, significado e identidad. Barcelona: Paidós.

Wertsch, J. V. (1993). Voces de la mente. Un enfoque sociocultural para el estudio de la acción mediada. Madrid: Visor.

Wilson, E. 0. (1989). Biofilia. México: Fondo de Cultura Económica. 